\title{
EPILEPSIA ABDOMINAL NA INFÂNCIA
}

\author{
Rubens Moura Ribeiro * \\ Jorge ARMBrust Figueiredo ** \\ VAleriana Moura Ribeiro*
}

A sindrome clínica caracterizada por manifestaçōes paroxísticas de localização abdominal acompanhada, ou não, de distúrbios de consciência, continua sendo diagnosticada com pouca freqüència. Embora, em 1907, Gowers ${ }^{9}$ já tenha descrito um complexo sintomático caracterizado por desconfôrto abdominal e náuseas, em pacientes com crises convulsivas, foi somente em 1941, com Klingman e col.14 que esta entidade pôde ser reconhecida em bases clinicas mais definidas. A origem cerebral das manifestaçōes abdominais paroxisticas foi demonstrada por Cushing ${ }^{3}$ e ulteriormente confirmada por Penfield e Jasper ", que estabelecaram a correlação diencefálica dessas manifestações clinicas. As alteraçōes elctrencefalográficas permitiram a Gibbs ${ }^{6}$ descrever, em 1951, certo tipo de disritmia cerebral considerada especifica da epilepsia abdominal.

Assim, é possivel formular o diagnóstico da epilepsia abdominal, caracterizado essencialmente pelo inicio paroxistico com sintomatologia abdominal e distúrbio de consciência, resposta favorável à terapêutica anticonvulsivante e alterações eletrencefalográficas $1,11,1 \pi, 16,17,18,20,22,23,24$.

O presente estudo foi feito com o intuito de analisar as manifestações clinicas e eletrencefalográficas de crianças portadoras de epilepsia abdominal. Pretendemos chamar a atençào para o valor da eletrencefalografia no diagnóstico das sindromes abdominais paroxisticas em crianças, principalmente pela pouca importância que geralmente se dá às suas queixas ou pelas dificuldades de seu reconhecimento.

Queremos salientar também que as anormalidades eletrencefalográficas são bastante freqüentes nesses facientes e que, portanto, nāo devemos poupar esforços por encontrá-las.

\section{MATERIAL E MÉTODO}

O material consta de 21 pacientes cujos dados de identificação figuram no quadro 1: 11 eram do sexo feminino e 10 do sexo masculino; as idades variaram entre 4 e 14 anos (para a seleçâo das idades foi utilizado o critério estabelecido por Stuart ${ }^{28}$ ).

Trabalho da Clinica Neurológica da Faculdade de Medicina de Ribeirāo Prêto, da Univ. de Sāo Paulo: *Assistente; ** Professor. 
As queixas clinicas referidas pelos pacientes puderam ser sumarizadas nas seguintes manifestaçôes de carăter jaroxistico: mal-estar indefinido (11 casos), movimentos no estômago (4), dores no estômago (3), sensações diversas no estómago (2), sensação intestinal (1).

$\mathrm{Na}$ medida do possivel, todos os pacientes foram submetidos a exames subsidiários de rotina, inclusive exame radiológico do abdome, no sentido de ser excluido processo que pudesse interferir com o diagnóstico neurológico. Em todos os casos houve resposta favorável à medicação anticonvulsivante, registrada em contrôles clínícos sucessivos.

Os registros eletrencefalográficos foram feitos em aparelho Grass de 8 canais, colocando os eletrodos conforme técnica usual internacional, com derivaçóes mono e bipolares. Utilizamos ativaçāo pela hiperpnéia em 17 pacientes e pelo sono espontān€o ou induzido por barbitúricos em 8 casos.

\section{RESULTADOS CLINICOS E ELETRENCEFALOGRAFICOS}

Da análise das manifestaçōes clinicas verificamos que apenas 4 pacientes (casos 6, 9, 17 e 18) não evídenciaram alteração de conscjência durante ou após a crise clínica. Após as manifestaçóes iniciais, 8 pacientes relataram crise convulsiva motora lateralizada (casos $3,9,10,11,14,16,20$ e 21); 4 apresentaram crises psicomotoras (casos 2, 7, 8 e 17); 3 tiveram crises do tipo grande mal (casos 4, 12 e 15); 2 tlveram crises do tipo ausência (casos 1 e 5 ).

Os traçados eletrencefalográficos foram normais em 5 pacientes (casos $3,4,{ }^{-} 10$, 12 e 16) e registraram alguma forma de alteração nos 16 restantes (76,2\%), sendo que, já durante o traçado de repouso, 7 pacientes evidenciaram EEG anormal (casos $6,7,8,11,13,14$ e 15 ). O exame durante o sono foi feito em 8 pacientes (casos

\begin{tabular}{|c|c|c|c|c|c|}
\hline Caso & Nome & Registro & Sexo & $\begin{array}{l}\text { Idade } \\
\text { (anos) }\end{array}$ & Resultados do EEG \\
\hline 1 & AMM & 194 & $F$ & 5 & 14 e $6 \mathrm{c} / \mathrm{seg}$ e onda sharp \\
\hline 2 & FFMJ & 1015 & M & 13 & 14 e $6 \mathrm{c} / \mathrm{seg}$ e onda sharp \\
\hline 3 & JEF & 169 & M & 14 & Normal \\
\hline 4 & $\mathrm{BL}$ & 19899 & M & 12 & Normal \\
\hline 5 & $\mathrm{DMC}$ & 2344 & $\mathbf{F}$ & & 14 e $6 \mathrm{c} / \mathrm{seg}$ e onda sharp \\
\hline 6 & $\mathrm{RCPC}$ & 14050 & $\mathbf{F}$ & 11 & Onda sharp \\
\hline 7 & LAO & 2871 & M & 6 & Onda sharp \\
\hline 8 & JASN & 7310 & M & 14 & Onda sharp \\
\hline $\mathrm{g}$ & WI & 22490 & $\mathrm{~F}$ & 10 & Onda sharp \\
\hline 10 & CGF & 15147 & $\mathbf{M}$ & 9 & Normal \\
\hline 11 & $\mathrm{JJ}$ & 2941 & $\mathrm{~F}$ & 7 & 14 e $6 \mathrm{c} / \mathrm{seg}$ e onda sharp \\
\hline 12 & $\mathrm{JCJ}$ & 2962 & M & 8 & Normal \\
\hline 13 & WRM & 2433 & M & 4 & Onda sharp \\
\hline 14 & $\mathrm{DHCC}$ & 25280 & $\mathbf{F}$ & 13 & Onda sharp \\
\hline 15 & MLL & 21434 & $\mathrm{~F}$ & 14 & Onda sharp \\
\hline 16 & MADS & 2886 & $\mathbf{F}$ & 12 & Normal \\
\hline 17 & $\mathrm{SM}$ & 3181 & M & 9 & Onda sharp \\
\hline 18 & $\mathrm{LCC}$ & 3167 & M & 7 & Onda sharp \\
\hline 19 & MES & 40818 & $F$ & 9 & Onda sharp \\
\hline 20 & AMM & 2434 & $\mathbf{F}$ & 12 & Onda sharp \\
\hline 21 & ALFC & 2773 & $\mathbf{F}$ & 12 & Onda sharp \\
\hline
\end{tabular}




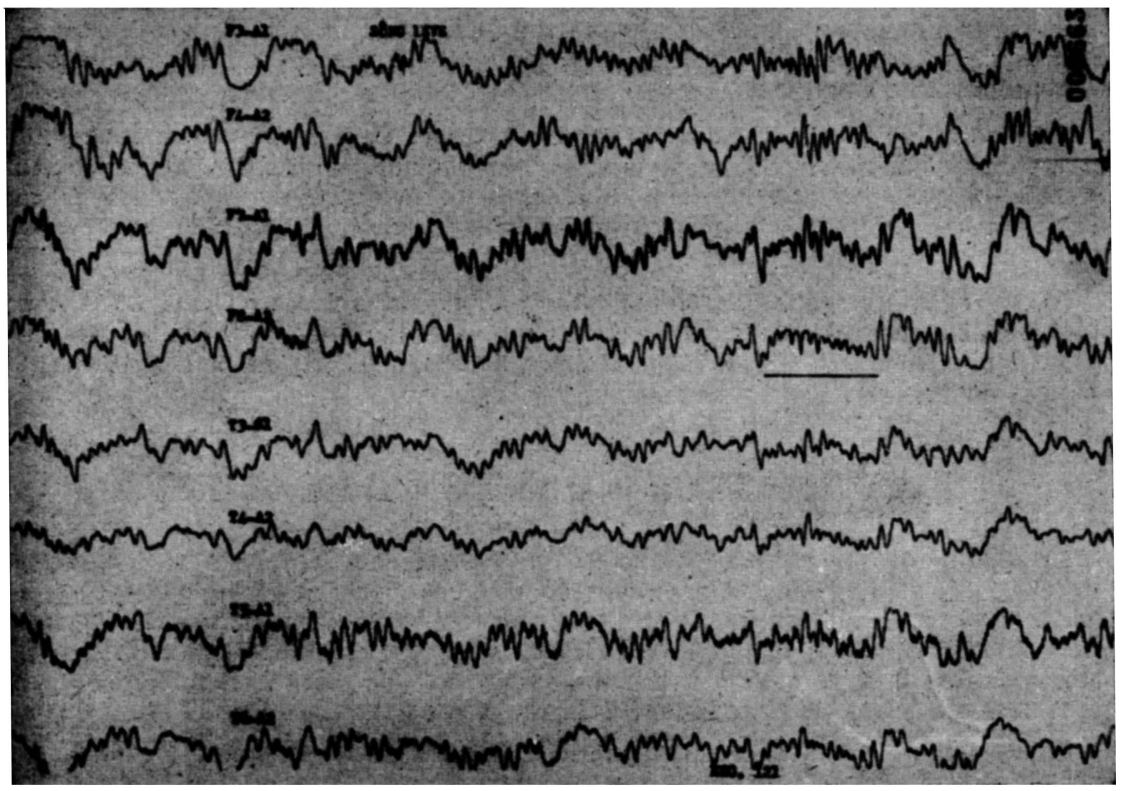

Fig. 1 - Caso 1 (EEG 121). Disritmia 14 ciclos por segundo em $F_{z}$.

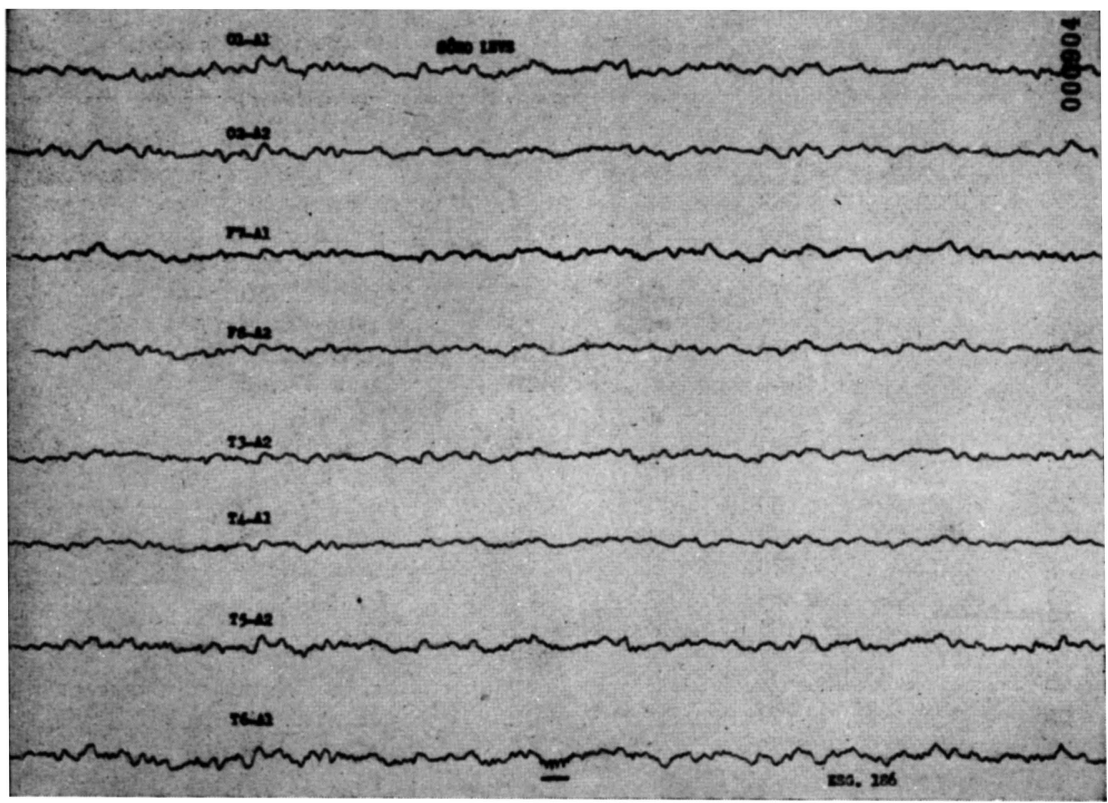

Fig. 2 - Caso 2 (EEG 186). Disritmia 6 ciclos por segundo em $T_{\text {. }}$ 


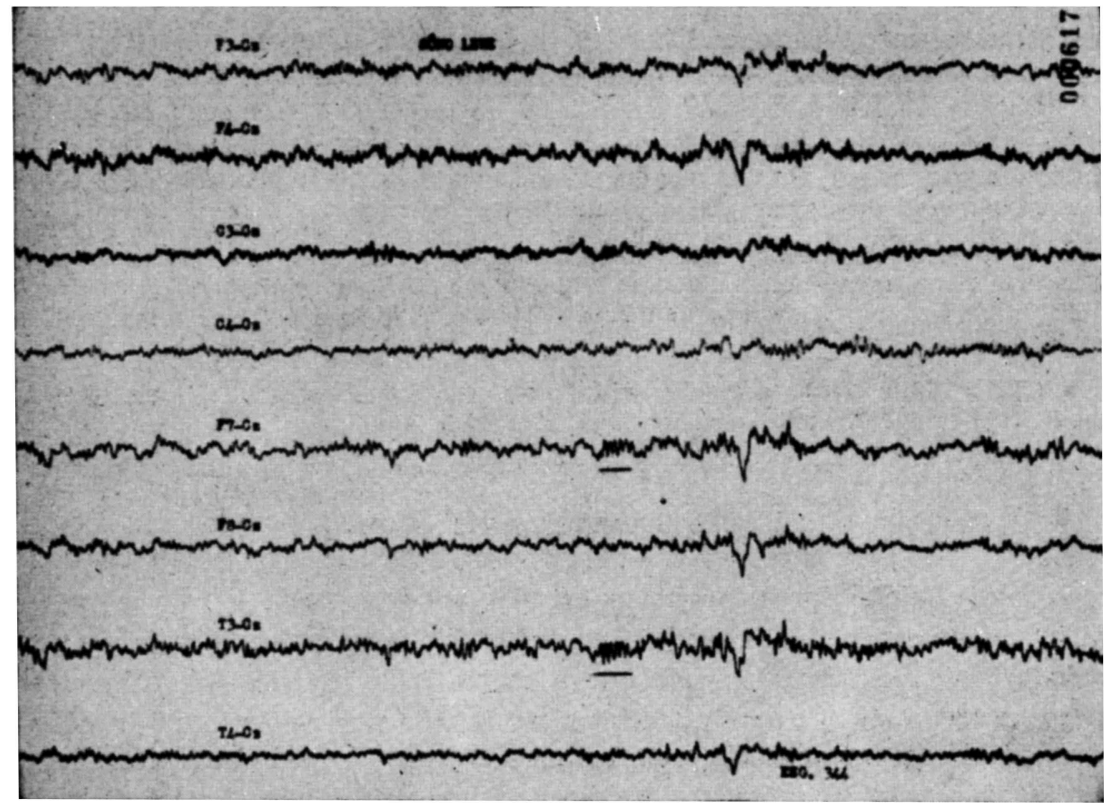

Fig. 3 - Caso 5 (EEG 344). Disritmia 6 ciclos por segundo em $F_{7}-T_{s}$.

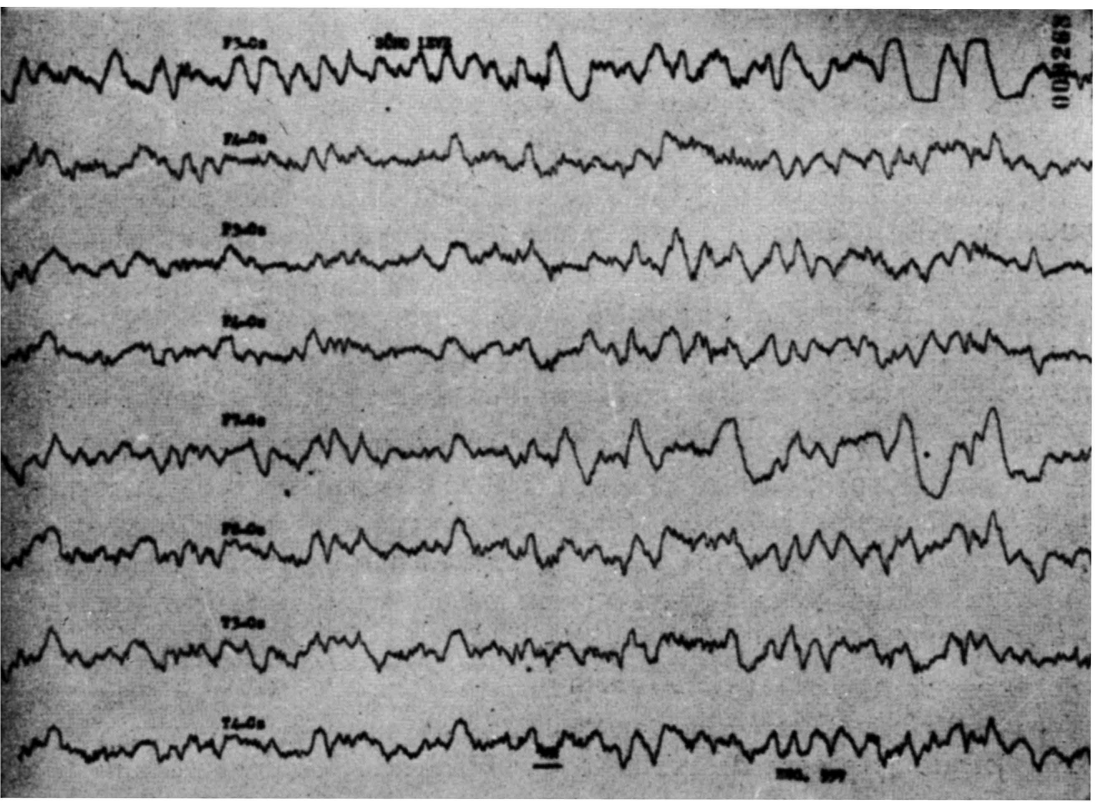

Fig. 4 - Caso 11 (EEG 559). Disritmia 6 ciclos por segundo em $T_{4}$. 
$1,2,3,5,9,11,13$ e 16), estando alterado em 5 ( $\operatorname{casos} 1,2,5,11$ e 13). A hiperpnéia, utilizada em 17 pacientes (casos $2,3,4,5,6,8,9,10,1214,15,16,17,18$, 19, 20 e 21), evidenciou alteração em 12 (casos $2,5,6,8,14,15,16,17,18,19$, 20 e 21).

Os resultados eletrencefalográficos (quadro 1) podem ser resumidos da seguinte maneira: disritmia por onda sharp (10 casos, ou seja 66,6\%); disritmia 14 e $6 \mathrm{p} / \mathrm{seg}$. ( 4 casos, ou seja 19\%); paroxismo p/onda delta ( 2 casos, ou seja 9,5\%).

A incidência topográfica das alteraçōes obedeceu a seguinte distribuicāo: foco subcortical no lobo temporal esquerdo (10 casos); foco subcortical no lobo temporal direito (4); foco cortical temporal médio esquerdo (1); foco cortical temporal médio direito (1).

Disritmia 14 e 6 por segundo evidenciada em 4 pacientes de 5 que apresentavam EEG alterado durante o sono (figs. 1, 2, 3 e 4).

\section{COMENTARIOS}

A contribuição fornecida pelos estudos experimentais e pela clínica demonstra a presença de centros de contrôle das funçōes autônomas no rinencéfalo e córtex cerebral ${ }^{5}$, sendo que a estimulação do giro hipocampal e do núcleo amigdalóide provoca alterações na função gastrintestinal ${ }^{4,}{ }^{7}$. Não há dúvida sôbre a importância que essas estruturas subcorticais têm para o contrôle das manifestaçōes ligadas ao aparelho digestivo ${ }^{8}$, seja pelos resultados experimentais registrados mediante eletrocorticografia, seja pela correlação da clínica com a eletrencefalografia ${ }^{12}$. Caracterizam-se êsses achados pela alta incidência de atividade epileptógena de projeção nos lobos temporais, indicando a estreita correlação entre as estruturas inferiores e a corticalidađe; êstes estímulos no córtex cerebral ativariam centros responsáveis pela regulação do tono e da atividade motora do trato gastrintestinal 25,28 .

A variabilidade de sintomatologia clínica evidenciada pelos nossos pacientes permite admitir a origem subcortical dessas manifestações, como demonstraram Green e Shimamoto ${ }^{10}$ quando estudaram a origem hipocampal da epilepsia abdominal. Por outro lado, nossos resultados eletrencefalográficos sugerem a interferência de mecanismos integradores do sistema nervoso central, seja pela alta incidência das descargas de origem subcortical, seja pela presença das disritmias 14 e 6 por segundo.

Representa, portanto, o EEG um método seguro para o diagnóstico diferencial entre uma síndrome clínica puramente abdominal daquela decorrente de patologia cerebral. Neste último caso o registro eletrencefalográfico evidencia em elevada percentagem alterações paroxísticas, na vigência ou não da manifestação abdominal ${ }^{2 i}$.

Apesar de Chao e col. ${ }^{2}$ e Kellaway e col. ${ }^{13}$ admitirem a remissão dos sintomas clínicos da epilepsia abdominal, com ou sem tratamento, ao contrário do que se espera da evolução das manifestaçōes convulsivas em geral, justifica-se a nosso ver a utilização de uma rotina cuidadosa com intuito de se analisar pelo menos o significado clínico dessas manifestações. 


\section{RESUMO}

São analisados do ponto de vista clínico e eletrencefalográfico, 21 casos relativos a crianças com epilepsia abdominal. Os autores chamam a atenção para as dificuldades que freqüentemente surgem para estabelecer êsse diagnóstico em crianças e, principalmente, para correlacioná-lo à uma patologia cerebral. Representa o EEG um exame subsidiário de valor como auxiliar no diagnóstico, principalmente quando são registradas as disritmias 14 e 6 por segundo. $\dot{E}$ considerada também a importância das estruturas subcorticais e suas ligações com o lobo temporal na regulação da atividade do trato gastrintestinal.

\section{SUMMARY}

\section{Abdominal epilepsy in children.}

The clinical and electroencephalographic finding of 21 children with abdominal epilepsy are analised. There is inquestionably an overlopping between the clinical syndrome of abdominal epilepsy and cerebral pathology. The EEG is usually abnormal and often shows the " 14 and 6 per second dysrhythmia" which is an indication of abdominal epilepsy. In basis of experimental and clinical evidence it seems probable that the state of activity of the gastro-intestinal tract is under cerebral cortex and lower centers influence.

\section{REFERENCIAS}

1. BARRAQUER-FERRÉ, L. - Abdominal epilepsy. Acta Psychiat. et Neurol. Scandinav., 29:71-77, 1954. 2. CHAO, D. H.-C.; DRUCKMAN, R. e KELLAWAY, P. - Convulsive Disorders of Children. W. B. Saunders Co., Philadelphia, 1958. 3. CUSHING, H. - Papers Relating to the Pituitary Body, Hyppothalamus and Parasympathetic Nervous System. Charles C. Thomas, Springfield (Illinois), 1932. 4. DLNLOP, C. W. - Viscero-sensory and somato-sensory representation in the rhinencephalon. Electroenceph. Clin. a. Neurophysioi., 10:297-304, 1958. 5. FULTON, J, F. - Physiology of the Nervous System, ed. 2. Oxford Lniversity Press, New York, 1943. 6. GIBBS, E. L. e GIBBS, F. A. - Electroencephalographic evidence of thalamic and hyppothalamic epilepsy. Neurology 1:136-144, 1951. 7. GLOOR, P. - Electrophysiological studies on the conections of the amygdaloid nucleus in the cat. Electroenceph. Clin. a. Neurophysiol., 7:223-242, 1955. 8. GLOOR, P. - The pattern of conduction of amygdaloid seizure discharge: an experimental study in the cat. Arch. Neurol, a. Psychiat., 77:247-258, 1957. 9. GOWERS, W. R. - The Borderland of Epilepsy. J. \& A. Churchill, London, 1907. 10. GREEN, J. D. e SHIMAMoTO, T. - Hyppocampal seizures and their propagation. Arch. Neurol. a. Psychiat., 70: 687-702, 1953. 11. HOEFER, P. F. A.; COHEN, S. M. e GREeley, D. M. - Paroxysmal abdominal pain: a form of epilepsy in chiltren. JAMA, 147:1-6, 1951. 12. JASPER, H. - Electroencephalography in Epilepsy and Cerebral Localization. Charles C. Thomas, Springfield (Illinois), 1941. 13. KELLAWAY, P.; CRAWLEY, J. W. e KAGAWA, N. - A specific electroencephalographic correlate of convulsive equivalent disorders in ehildren. J. Pediat., 55:582, 1959. 14. KLINGMAN, W.; LANGFORD, W. S.; GREElEY, D. M. e HOEFER, P. F. A. - Paroxysmai attacks of abdominal pain: epileptic equivalent in children. Tr. Am. Neurol. Ass., 67:228-229, 
1941. 15. LIVINGSTON, S. - Abdominal pain as a manifestation of epilepsy (abdominal epilepsy) in children. J. Pediat., 38:687-695, 1951. 16. MILLICHAP, J. G.; LOMBROSO, C. T. e C. T. LENNOX, W. G. - Cyclic vomiting as a form of epilepsy in children. Pediatrics 15:705-714, 1955. 17. MULDER, D. W.; DALY, D. e BAILEY, A. A. - Visceral epilepsy. Arch. Int. Med., 93:481-493, 1954. 18. NAEYE, R. L. - Cyclic tever abdominal pain and grand mal seizures: case report. Ann. Int. Med., 48:859-963, 1958. 19. PENFIELD, W. - Epilepsy automatism and the centreencephalic integrating system. A. Res. Nerv. Ment. Dis. Proc., 30:513-528, 1952. 20. PENFIELD, W. e JASPER, H. - Epilepsy and Cerebral Localization. Charles C. Thomas, Baltimore, 1941. 21. PENFIELD, W. e JASPER, H. - Epilepsy and the Functional Anatomy of the Human Brain. Little Brown Co., Boston, 1954. 22. PRICHARD, J. D. - Abdominal pain of cerebral origin in children. Canad. M. A. J., 78:665-668, 1958. 23. SHEEBY, B. N.; LITTLE, S. C. e STORE, J. J. - Abdominal epilepsy. J. Pediat., 56:355-363, 1960. 24. SNYDER, C. H. - Epileptic equivalent in children. Pediatries 21:308-318, 1958. 25. SPIEGEL, E. S.; WESTON, K. e OPPENHEIMER, M. J. - Posimotor foci influencing the gastrointestinal tract and the descending pathways. J. Neuropath. a. Exper. Neurol., 2:45-53, 1943.26. STUART, H. C. - Normal growth and development diving adolescence. New England J. Med., 234:666, 1946. 27. van BUREN, J. M. - The abdominal aura: a study of abdominal sensations occurring in epilepsy and produced by depth stimulation. Electroenceph. Clin. a. Neurophysiol., 15:1-19, 1963. 28. WATTS, J. W. e FULTON, J. A. - Intussusception: the reiation of the cerebral cortex in intestinal motility in the monkey. New England J. Med., 210:833, 1934.

Clinica Neurológica, Faculdade de Medicina - Ribeirão Prêto, Săo Paulo - Brasil. 